Referencia para citar este artículo: Ronzón-Tirado, R. C.; Yedra, L. R. \& González-Flores, M. del P. (2017). Modelos parentales y su relación con la violencia en las parejas del mismo sexo. Revista Latinoamericana de Ciencias Sociales, Niñez y Juventud, 15 (2), pp. 1137-1147. DOI:10.11600/1692715x.1522317062016

\title{
Modelos parentales y su relación con la violencia en las parejas del mismo sexo*
}

\author{
Rosa CAROLINa RonzóN-TiRADo ** \\ Psicóloga en Ahteca, México. \\ LUIS REY YEDRA *** \\ Profesor-Investigador Universidad Veracruzana, México. \\ MARÍA DEL PILAR GONZALEZ-FLORES ***** $^{\text {* }}$ \\ Profesora-Investigadora Universidad Veracruzana, México.
}

\begin{abstract}
Artículo recibido en junio 17 de 2016; artículo aceptado en septiembre 12 de 2016 (Eds.)
\end{abstract}
- Resumen (descriptivo): este artículo forma parte de una investigación más amplia cuyo objetivo fue describir los elementos relacionados con la violencia en parejas gays y lésbicas. Abordamos los resultados sobre la relación de los modelos parentales con la violencia ejercida al interior de estas y otras relaciones interpersonales. Seguimos un método descriptivo cualitativo y utilizamos grupos focales para recopilar la información, en donde participaron ocho hombres gays, seis mujeres lesbianas y una mujer bisexual, reclutados por disponibilidad. Encontramos que presenciar violencia entre los padres o ser expuestos a experiencias tempranas de maltrato destacan como principios favorecedores de futuras dinámicas violentas de pareja; y que el rechazo parental tras "salir del closet", junto con la dificultad para distinguir la violencia fuera del contexto heterosexual familiar, son obstáculos para detectar y afrontar la violencia.

Palabras clave: violencia familiar, maltrato infantil, relaciones interpersonales (Tesauro de Ciencias Sociales de la Unesco).

Palabras clave autores: violencia en la relación de pareja, pareja del mismo sexo, gays, lesbianas, bisexuales.

\section{Parental models and their relation to violence among same-sex couples}

- Abstract (descriptive): this article is part of a wider study that has the objective of describing the elements related to violence among gay and lesbian couples. The authors analyze the results of looking at the relation between the parental model and violence inflicted within these and other

\footnotetext{
* Este artículo de investigación científica y tecnológica se deriva de un estudio más amplio denominado "Violencia en las relaciones de pareja del mismo sexo", elaborado como tesis de maestría en Desarrollo Humano, Universidad Veracruzana. Xalapa, Veracruz, México. Acta de Colegio de Profesores de la Maestría en Desarrollo Humano 100614 del Instituto de Psicología y Educación de la Universidad Veracruzana. Este artículo presenta los resultados de la investigación finalizada llevada a cabo entre el 12 de agosto de 2014 y el 20 de enero de 2016. Área de conocimiento: Psicología; Subárea de conocimiento: Psicología.

** Maestra en Desarrollo Humano, Universidad Veracruzana. Orcid: 0000-0003-0295-242X. Correo electrónico: rc.ronzon@gmail.com

*** Postdoctor en Psicología, Universidad Autónoma de Lisboa. Doctor en Orientación y Desarrollo Humano, Universidad Iberoamericana Ciudad de México. Orcid: 0000-0002-5557-4146. Correo electrónico: lyedra@uv.mx

**** Postdoctora en Psicología, Universidad Autónoma de Lisboa. Doctora en Educación, Universidad Nacional de Educación a Distancia. Madrid, España. Orcid: 0000-0002-9991-7243. Correo electrónico: pgonzalez@uv.mx
} 
interpersonal relationships. We used a qualitative descriptive method and focal groups to collect information with participation from 8 gay men, 6 lesbian women and 1 bisexual woman, who were selected based on their availability. The authors concluded that witnessing violence between parents or being exposed to early violent experiences were significant factors in encouraging violent dynamics within. In addition, parental rejection after "coming out of the closet" along with the difficulty with identifying violence outside of the generally accepted heterosexual family context were obstacles for identifying different types of violence.

Key words: family violence, child abuse, interpersonal relationships (Social Science Unesco Thesaurus).

Authors key words: couple relationship violence, same-sex couples, gay, lesbian, bisexual.

\section{Modelos parentais e sua relação com a violência em casais do mesmo sexo}

- Resumo (descritivo): este artigo é parte de uma pesquisa mais ampla cujo objetivo foi descrever os elementos relacionados com a violência em casais homoafetivos. Abordamos os resultados da influência dos modelos parentais sobre a violência nestes relacionamentos e outras relações interpessoais. Seguimos um método descritivo qualitativo e utilizamos grupos focais como técnica de coleta de informaçõe. Participaram 8 homens gays, 6 mulheres lésbicas e 1 mulher bissexual. Descobrimos que testemunhar a violência entre os pais ou ser expostos a experiências precoces de abuso posicionaram-se como elementos que provocaram dinâmicas violentas entre eles; e que a rejeição dos pais depois de "sair do armário", juntamente com a dificuldade que eles experimentaram para distinguir a violência fora do contexto heterossexual familiar foram os obstáculos para detectar a violência.

Palavras-chave: violência familiar, abuso infantil, relações interpessoais (Thesaurus de Ciências Sociais da Unesco).

Autores palavras-chave: violência na relação, parceiro do mesmo sexo, gays, lesbicas, bissexual.

-1. Introducción. -2. Método. -3. Participantes. -4. Resultados. -5. Discusión. -Lista de referencias.

\section{Introducción}

La violencia en las parejas de personas gays, lesbianas y bisexuales, al igual que en cualquier otra relación, se presenta como una situación de abuso de poder o control de un miembro sobre el otro mediante distintos ataques de tipo psicológico, físico o sexual. A pesar de su existencia, esta problemática ha sido poco estudiada y velada por la prioridad conferida -en países de Latinoamérica y específicamente en México- a la investigación, intervención y prevención del abuso en relaciones heterosexuales.

Su escasa visualización, aunada a los mitos y desconocimiento de las sexualidades distintas a la heterosexual, ha implicado que ni siquiera las personas que sufren este tipo de violencia estén dispuestas a reconocerla, pues consideran que ello daría motivo para ser discriminadas.
Este temor latente al rechazo junto con otros factores como la opresión de grupos políticos y la escasez de datos estadísticos, son algunos de los obstáculos a los esfuerzos realizados hasta la fecha para la detección y abordaje específico de este fenómeno.

Si bien la literatura sobre este tema empieza a ser más extensa, sobre todo en cuanto a su incidencia (Aldarte, 2010; Gil, Machado \& Atunes, 2006; Mak, Chong \& Kwong, 2010; Reyes, Rodríguez \& Malavé, 2005), y la influencia de los estresores minoritarios como la homofobia -consciencia de estigma y heteronormatividad para desarrollo de la violencia (Carvalho, Lewis, Derlega, Windstead \& Viggiano, 2011; Marín, 2009)-, dentro de este rubro de investigaciones aún son pocos los trabajos en los que además se abre la discusión sobre la relación con otros factores "comunes" a otros grupos poblacionales, 
como el consumo de sustancias, los estilos de resolución de conflictos, la escasez de redes de apoyo y la replicación de modelos familiares comportamentales, siendo este último elemento sobre el cual centramos la atención en este artículo, debido a la notable relación que manifestaron los participantes entre la violencia que vivieron en sus familias y las experiencias tempranas de maltrato con la violencia en sus relaciones de pareja y en otras relaciones interpersonales significativas.

Al ahondar sobre este tema, nuestro objetivo es contribuir a los hallazgos encontrados previamente por otros investigadores e investigadoras sobre el aprendizaje de la violencia en el hogar de origen, y sobre cómo ésta se relaciona con la exposición temprana a experiencias de maltrato y a dinámicas caracterizadas por el abuso del poder o la fuerza (López \& Ayala, 2011; Padilla, 2015; Toro-Alfonso \& Rodríguez-Madera, 2003; Vargas, 2003). Además buscamos generar un antecedente sobre el papel que supone la familia para este grupo poblacional al momento de enfrentar una situación de violencia con sus parejas, pues tal como lo señalan Perucchi, Coelho y Dos Santos (2014), la familia, más que representar un soporte para hacer frente a la violencia, puede significar un sostén de legitimación para la misma, por el rechazo posterior al externar su orientación sexual o "salir del closet", que lleva frecuentemente a la ruptura del vínculo y a la expulsión familiar.

Cabe mencionar que en ninguno de los estudios que encontramos en la revisión literaria sobre la violencia en las parejas de la comunidad gay, se ahonda sobre la influencia percibida de los repetitivos mecanismos conflictuales familiares desde el discurso propio de los individuos, pues los resultados expuestos se limitan a ser enunciativos o estadísticos (Aldarte, 2010; ToroAlfonso \& Rodríguez-Madera, 2003). Así, este artículo es un esfuerzo para abordar el mundo fenomenológico de la experiencia de violencia en las relaciones de pareja del mismo sexo y su relación con los modelos parentales vividos en el hogar de origen, desde la experiencia propia de los actuantes, situación de la que, en muchas

1 Como se denomina coloquialmente al hacer pública una orientación sexual distinta a la heterosexual.

Rev.latinoam.cienc.soc.niñez juv 15 (2): 1137-1147, 201 http://revistalatinoamericanaumanizales.cinde.org.co

DOI:10.11600/1692715x.1522317062016 ocasiones, ha sido privada esta comunidad al ser estudiada desde una visión heteronormada e incluso patologizante (Castañeda, 2011).

Para realizar esta labor, partimos del supuesto de que la familia, más allá de cualquier característica que posean sus integrantes, representa el eje central del ciclo de vida a través del cual trascurre la existencia de las personas, como la célula social que en el interior acoge a sus miembros y a su vez los relaciona con el exterior y con otros organismos similares (Estrada, 2014), ya que en ella se vive un entramado de actitudes, valores, comportamientos, normas y creencias que tienen una fuerte influencia sobre el comportamiento de sus integrantes, sobre su desarrollo social, físico, afectivo e intelectual, lo que le lleva a ser en sí misma un elemento de salud o de origen de diversos problemas (Prieto-Montoya, Cardona-Castañeda \& VélezÁlvarez, 2016).

A modo de pequeña sociedad, la familia se posiciona como un medio en el que, en el mejor de los casos, se permite toda clase de ensayos y fracasos, en un ambiente de protección, amor incondicional, tolerancia y firmeza; sin embargo, muchas veces en el interior de las familias no se logra consumar estas tareas y se viven modelos parentales disfuncionales, en los que se carece de la guía, estabilidad y congruencia necesarias para el desarrollo de los miembros. Esta dinámica genera poco a poco temores, creencias erróneas y sentimientos de minusvalía en las personas, que perpetúan los comportamientos desadaptativos y la construcción de relaciones conflictivas o violentas en las cuales experimentan los mismos sentimientos que vivieron en sus familias de origen (Canales, 2014).

\section{Método}

Tal como lo mencionamos anteriormente, este artículo forma parte de una investigación más amplia que lleva por título "Violencia en las relaciones de pareja del mismo sexo", cuyo objetivo principal fue describir los elementos asociados con la violencia en las relaciones gays y lésbicas. Para la construcción del conocimiento seguimos un método descriptivo de corte cualitativo, debido a que se posicionaba 
como el más adecuado para acceder al nivel de los discursos y significados de los sujetos participantes en los que quedaron expresadas las razones subjetivas de la acción social y su comportamiento (Rubio \& Varas, 2004).

Para recopilar la información llevamos a cabo dos grupos focales, metodología definida por Delgado y Gutiérrez (1995) como una técnica que trabaja con el habla mediante la articulación de lo subjetivo con lo grupal, y que hace hincapié en el discurso social diseminado en el intercambio de palabras, gestos $y$ acciones. Nuestro propósito principal al usar esta técnica fue propiciar el surgimiento de actitudes, sentimientos, creencias, experiencias y reacciones de los sujetos participantes (Escobar \& Bonilla-Jiménez, 2009) sobre la violencia en las relaciones de pareja gays y lésbicas, así como los elementos involucrados en su incidencia. Para nosotros, esto no hubiese sido fácil de lograr con otras técnicas de corte cualitativo, tales como la entrevista individual o grupal, pues ésta, a diferencia de las demás, nos permitió recopilar mayor información en menor tiempo; implicó la ventaja de brindar no sólo los datos emitidos verbalmente, sino la información relativa a la forma en que lo decían junto con la interacción entre los individuos participantes durante las reuniones; además nos permitió aprovechar la flexibilidad inherente a dicha interacción para realizar dos grupos en lugar de uno solo, con el fin de alcanzar el mayor grado de saturación de datos hasta que la recolección de información pareciera ya no tener un valor interpretativo adicional (Onwuegbuzie, Dickinson, Leech, \& Zoran, 2011).

Grabamos las sesiones de los grupos en audio y video, y posteriormente las transcribimos a texto. Una vez que realizamos esta tarea, proseguimos al análisis del contenido de los datos recabados y a la categorización manual de las unidades de registro identificadas para cada elemento; en esta labor, tal como lo mencionan Quilaqueo y San Martí (2008), nos apoyamos en la codificación abierta del discurso, basados en la teoría revisada y a criterio del equipo de investigación. No recurrimos al uso de algún paquete estadístico, pues el hecho de que fueran
15 participantes nos facilitó realizar esta tarea de manera manual.

Los temas abordados durante las sesiones fueron estructurados previamente en una guía de grupo focal, validada y ajustada a partir de la revisión hecha por dos expertas en el estudio $e$ investigación de relaciones interpersonales que pueden dejar al descubierto distintos tipos de violencia; los primeros tópicos abordados estuvieron más relacionados con la vivencia de la orientación sexual y el establecimiento de las relaciones de pareja, y los últimos temas estuvieron más orientados a la experiencia actual de violencia en las parejas y a los antecedentes de violencia en la familia de origen. Esto, con la intención de pasar de la información más general a la más específica sobre el tema, y de lo menos comprometedor a lo más confidencial.

Las principales funciones de la moderadora de las sesiones, siguiendo lo señalado por Rubio y Varas (2004), fueron la provocación inicial, la puesta en marcha y el mantenimiento de la discusión y de la dinámica grupal, buscando que se trascendiera la dinámica de preguntarespuesta entre moderadora y participantes, y se desarrollara así un proceso de interacción grupal que caracteriza esta técnica. Esta labor fue llevada a cabo manteniendo presente que la provocación del discurso no solo se centraba en conductas explícitas -como los señalamientos verbales-, sino cuidando siempre tanto el contenido como el proceso grupal.

\section{Participantes}

Formó parte de este estudio una muestra por conveniencia no probabilística, reclutada acorde con su disponibilidad de tiempo y con su deseo de colaborar en la investigación, la cual estuvo conformada por 15 personas que cumplieron con dos criterios de inclusión: a) tener una relación de pareja al momento del estudio o haberla tenido hasta hace un año atrás con una persona de su mismo sexo, y b) asumirse como una persona gay, lesbiana o bisexual. Del total, ocho personas fueron hombres gays, seis mujeres lesbianas y una mujer bisexual, con un rango de edad de entre 19 y 27 , y un promedio de 23 años. Cinco de los participantes vivían con su pareja, cinco vivían 
con sus familias de origen y los cinco restantes solos; cinco contaban con estudios concluidos de bachillerato, ocho de licenciatura y dos de posgrado. Los 15 individuos participantes residían al momento de la entrevista en la ciudad de Xalapa, Veracruz, México; trece de ellos tenían una relación de pareja al momento en que se llevó a cabo el estudio y los dos restantes la habían tenido en los seis meses anteriores a su participación.

El reclutamiento de los sujetos participantes fue complicado debido a la temática abordada, que resultó amenazante para algunos por el hecho de asumir simultáneamente su orientación sexual y el experimentar violencia en sus relaciones, por lo que tuvimos que recurrir a tres medios para contactarlos: la convocatoria por medio de Internet en redes sociales de temática homosexual, el contacto con asociaciones civiles, y la técnica de bola de nieve en la que una vez que localizábamos a una primera persona que cumpliese los requisitos para participar, le pedíamos contactar a otras personas potencialmente participantes, y a éstos, a su vez, les solicitábamos nuevos nombres.

\section{Resultados}

Al igual que en estudios previos realizados sobre violencia en las relaciones de pareja lésbicas y gays (Vargas, 2003; Toro-Alfonso \& Rodríguez-Madera, 2003; López \& Ayala, 2011; Molina, Echeverría \& Henseleit, 2014), las personas participantes identificaron que el estar expuestas desde la niñez a modelos parentales disfuncionales o violentos había favorecido que ellas lo viesen como una forma "normal" -deseable o indeseable- de actuar para relacionarse, ya que las prácticas de control, manipulación e inclusive las agresiones físicas, podían ser concebidas como un medio para solucionar conflictos, como una manera de comunicarse y como una forma de demostrarse amor o interés. Esto era válido, tanto en las situaciones en las que las dinámicas violentas fuesen vistas en la relación de pareja de sus padres y madres, y de su familia extensa -mediante juegos de poder, chantajes, control, intimidación, descalificación, amenazas,

Rev.latinoam.cienc.soc.niñez juv 15 (2): 1137-1147, 2017

http://revistalatinoamericanaumanizales.cinde.org.co

DOI:10.11600/1692715x.1522317062016 insultos, gritos, y en cuatro casos golpes-, como cuando habían vivido experiencias tempranas de maltrato, tales como abusos u omisiones por medio de chantajes, amenazas, descalificación, insultos, gritos, situaciones de indiferencia o de invisibilidad, y en uno de los casos condiciones de violencia física constante.

Como principales patrones de comportamiento disfuncionales modelados por sus padres y madres, señalaron que en sus familias se reprendía el externar los sentimientos de manera abierta, honesta o auténtica, que se condicionaba el amor a permanecer bajo ciertos estados de ánimo inexpresivos o aplanados, y que la resolución de conflictos podía, o bien ser evasiva -pues no se llegaba a la aclaración del desacuerdo, ni se daba pauta al acercamiento de las necesidades del otro-, o agresiva, mediante la distribución inequitativa del poder y la ejecución de comportamientos controladores o violentos de tipo psicológico o físico.

Presentamos el análisis de los datos obtenidos agrupados en tres categorías: violencia presencial entre sus padres, experiencias tempranas de maltrato, e impacto percibido en otras relaciones interpersonales. A modo de ejemplo y para dar mayor claridad, exponemos algunas de las unidades de registro extraídas del discurso de los sujetos participantes para cada caso. Al final de cada registro mencionamos entre paréntesis el nombre, el sexo y la edad del individuo participante que aportó el comentario. Los nombres han sido cambiados por cuestión de privacidad.

\section{Violencia presencial entre los padres}

"Sí hubo mucho, mucho maltrato o agresión, este... mental y verbal, tal vez no física pero hasta cierto punto yo debo aceptar que a mí me tocó ver mucho, porque a mí, toda mi inocencia, mi infancia... yo me la pasé viendo a mi mamá, en vez de estar jugando conmigo, estar sufriendo... Entonces en mí sí influyó en cómo soy ahora con mi pareja, que hemos llegado hasta los golpes... es doloroso, ¿no? Porque bueno, en mi caso, es de la persona que más me he enamorado y digo, si es de la 
que más me he enamorado ¿como por qué le hice esto?" (Ramón, H, 19 años).

Al analizar el discurso de las personas participantes, encontramos que para 11 de ellas el hecho de haber visto violencia entre sus padres y madres se posiciona como una pauta de comportamiento que, si bien vivieron como indeseable, la siguen replicando en sus relaciones posteriores, al grado de que dos participantes llegaban a considerarle como apreciable por su relativa eficacia: "Si así lo hacían mis papás y ya tengo 19 y sigo vivo, pues igual y es una buena solución" (Pedro, $\mathrm{H}$, 19 años).

Para tres, el rechazo hacia lo que vivieron es tal que se han propuesto no actuar como sus padres y madres, con el fin de no continuar replicando los patrones comportamentales que les desagradan; sin embargo, en cuanto hay un mayor involucramiento en la relación, los modelos violentos surgen en su actuar, siendo esta una situación que les genera un malestar aún mayor.

"En mi casa por ejemplo no... no hubo violencia física, pero mi papá, tiene un carácter muy fuerte, y yo por ejemplo llegué a decir, no pues lo que no me gusta de mi papá, yo no lo voy a hacer, lo que a mí no me gusta de mi papá, nunca lo voy a hacer... pero cuando menos te das cuenta, y estás teniendo una relación... estás comportándote igual que tu papá, $y$ te lo dejan ver o te lo dicen y cuando "te cae el 20", te sientes peor" (Óscar, H, 25 años).

Llama la atención que para algunos participantes el hecho de evitar la violencia que vieron durante su niñez cobra vital importancia, sobre todo cuando se percatan de que sus hermanos y hermanas vivían situaciones similares a las de sus padres y madres, lo cual consideran que acrecienta el riesgo de "ser violentos", asumiendo una visión estática y determinista de la realidad, ante la cual llegan a experimentar temor sobre la inminente replicación de conductas violentas que ejecutarían si se descuidan o pierden el control.

"Mi hermana sí lo es (violenta) en su relación, y mi hermano lo es pero con las demás personas, él es de, de... se pelea y lastima mucho, fisicamente ¿no? O sea, tiene problemas de violencia y yo pienso que es porque vio mucha violencia de niño ¿no?, pero yo al contrario... pero pienso que... si yo fuera una persona violenta, si me atreviera a serlo, lo sería en exceso, entonces... yo creo que por eso me controlo, temo ser así... y me paso de pasiva, por lo mismo supongo" (Esther, M, 27 años).

Más de un participante mencionan que, en el intento de evitar ser como sus padres y madres en una relación y diferenciar sus yo-mismo de las propias familias de origen, optaron por su legitimación y por volverse indiferentes ante la violencia. Si bien ello genera un supuesto bienestar en un principio -por no ser "iguales" a sus madres y padres-, la disfunción en las relaciones que establecen vuelve a aparecer posteriormente, pues la indiferencia continúa cumpliendo una función en un escenario más amplio, en el que la persona no actúa conforme a la valoración de sí mismo, sino en contraposición a los modelos parentales que vivió (Bowen, 1991), resultando en una nueva relación violenta en donde quizá ya no juegue tanto un rol de individuo victimario pero sí contribuye a la perpetuación del problema.

Cabe mencionar que, si bien la reproducción de la violencia a partir de aprendizajes tempranos resulta un hecho de fácil identificación para algunos sujetos participantes, para otros no lo fue tanto, pues los embates, al llevarse a cabo fuera del contexto heteronormado familiar, son considerados conductas inofensivas entre dos personas "similares por ser del mismo sexo", y dejan de ser valorados como violencia al no encajar en estereotipos donde la mujer es concebida como víctima y el hombre como victimario (Padilla, 2015). Así, los significados y atributos que han interiorizado de los modelos de comportamiento parental sobre los roles de género, homofobia y heteronormatividad, contribuyen no solo a replicar la violencia sino a perpetuarla, por la incongruencia entre el cómo viven los embates y lo que creen sobre ellos. "Es justificable que... entre dos personas del mismo sexo se puedan agredir porque son iguales... son dos mujeres, pero que un hombre me toque jamás" (Karla, M, 26 años). 


\section{Experiencias tempranas de maltrato}

"Me hizo enojar tu papá, me voy a desquitar contigo ¿no?, entonces eso si influyó demasiado, influyó demasiado en mi conducta porque suelo ser callado, porque yo recuerdo que si respondía a mi madre era golpe seguro ¿no?... Me llegó a marcar las piernas con cables, de los golpes, pero bueno pues todo eso, a mí como que hasta cierto punto dije bueno, el hablar no es bueno, me voy a quedar callado, voy a reprimir todo eso que siento" (Pedro, H, 19 años).

Respecto de las experiencias tempranas de maltrato, identificamos que ocho de los individuos participantes mencionaron que el haber sido expuestos a maltrato físico y psicológico por parte de sus padres y madres -especialmente por parte de la madre-, había favorecido que aprendieran a comportarse de manera sumisa, que tuvieran procesos comunicativos poco efectivos, y que naturalizaran distintas prácticas de poder o de control como formas de relación aceptables a cambio de la aprobación o protección que su padre o su madre les pudiese propiciar durante la infancia, pues de no actuar conforme a lo que se les demandaba eran rechazados o maltratados.

Así, tras la sucesiva exposición a modelos comportamentales caracterizados por la escasa empatía, por la incongruencia y por una valoración condicional, las personas participantes aprendieron que sus valores, opiniones o sentimientos tenían poco valor en comparación con lo que sus padres necesitaban. Si bien, en un principio esta dinámica era propia de la relación padre/madre-hijo/hija, cinco de los ocho individuos que habían experimentado maltrato al haber crecido tienden a replicar dichos comportamientos en sus relaciones subsecuentes.

"Quizá no es tanto como violencia, pero de que te tienes que quedar callado, reservado, hasta cierta forma, realmente lo sigues aplicando en años después, de que tú por ser niño tienes que escuchar a los adultos, ellos están bien y tú... si no estás con ellos, estás mal; entonces te vas quedando con tantas cosas y ya cuando estás con alguien en algo muy íntimo, luego salen esas cosas; es cuando creo estás más expuesto, cuando estás más vulnerable, y... todo eso sale a flor de piel... es muy raro porque hasta cierto punto piensas que ese eres tú" (Fausto, H, 24 años).

Las rutinas y patrones de comportamiento aceptados en la estructura familiar de la que provienen, sin importar si se trata de comportamientos violentos físicos $\mathrm{o}$ psicológicos, son vistos como algo cotidiano y normal, lo cual contribuye a su legitimación al interactuar con su pareja, pues si ya han vivido algún tipo de maltrato o violencia con su madre o padre, experimentarlo nuevamente en sus relaciones no representaba motivo de sobresalto.

"Más que nada, las cosas de manipulación de mi mamá, algunas las he replicado, y... también soportar otras ¿no?... que soportaba en mi mamá, he soportado en mis parejas" (Karla, M, 26 años).

En dos de los casos, hallamos que la ineficacia de los procesos comunicativos de los padres y las madres, y las escasas habilidades para la resolución de conflictos de éstos, favorecen que asuman a su hijo o hija como intermediario o confidente con quien hablar acerca de sus problemas maritales; en dichas familias se triangulan las relaciones afectivas y los sujetos participantes pasan desde pequeños a formar parte de los bloques enfrentados (Segura, Gil \& Sepúlveda, 2006). Esto ocasiona que los hijos e hijas tomen partido en el conflicto, asuman los comportamientos disfuncionales como normales o "cercanos" y posteriormente tiendan a reproducir las disputas que han visto en sus mayores.

"Resulta que cuando tengo 18 años, mis papás empiezan con un conflicto por el cual se van a divorciar y todos los problemas me los venían a decir a mí. Entonces como yo era la mayor tenía que resolver todos sus conflictos, y fue así de ¡qué...! entonces en algún punto creo que por eso en mis dos primeras relaciones, yo como que aceptaba la 
violencia, porque veía como que el círculo familiar más cercano" (Ana, M, 21).

Finalmente, identificamos como característica que perpetúa la violencia en estas parejas el escaso aprecio, protección o amor percibido por algunos de los individuos participantes por parte de su padre o madre durante la infancia -en uno de los casos por presentar comportamientos "propios del otro sexo"-, y especialmente en la adolescencia cuando decidieron externar su orientación sexual o "salir de closet". Esta situación favoreció, en cuatro de los casos en estudio, la ruptura del vínculo familiar, el aislamiento progresivo de amistades, y que la principal o única persona de la que recibían apoyo o contención emocional fuese la pareja de la cual dependían emocionalmente; esto, a su vez, dio pauta a que se pasaran por alto los episodios violentos o que se les asumiera como muestras de afecto.

"Llegué así de... oye mamá te quiero presentar a alguien... llevábamos un mes saliendo... sí, entonces a mi mamá, como que se le vino el mundo encima, y hasta me corrieron de la casa y fue que me pasé a vivir con él y toda la onda ... y entonces como que se acentúa más este... tu necesidad, yo creo de que la persona esté contigo pues..., dices, no te voy a dejar así nada más jno!... Tu mente se pone a... volar y dices $y . .$. dónde andará, qué estará haciendo" (Pedro, H, 19).

\section{Impacto percibido sobre otras relaciones significativas}

Sobre el impacto percibido de los modelos parentales disfuncionales en las demás relaciones interpersonales, identificamos que al igual que con sus parejas, los sujetos participantes tienden a reproducir comportamientos desadaptados o poco efectivos que vivieron en sus familias de origen, pues si el padre o la madre fue una persona manipuladora o violenta, simplemente le "fotocopian" su actuar y, a partir de ello, se relacionan con sus amistades o conocidos.

Esto es igual tanto para los hombres como para las mujeres que participaron, pues en nuestros resultados encontramos que ambos sexos aprenden a ser violentos, sin importar el sexo del individuo progenitor que modelara la violencia -cabe mencionar que nuevamente encontramos que las madres tienden a ser vistas como más violentas).

"Mi mamá es toda explosiva, y yo así se lo fotocopié... yo tengo, mmm... ansiedad, y también, soy hiriente, no de golpear, nunca... pero sí, si estoy como que ansioso y me están provocando y vuelven a hacer lo mismo, lo mismo, lo mismo y como que no se están dando cuenta... si están pique, pique, pique... soy igual, hiriente" (Jorge, H, 24 años).

En algunos casos, los comportamientos se extienden a todos los espacios donde interactúan, pues los déficits en el funcionamiento interpersonal se presentan no sólo en aquellas relaciones significativas, sino en encuentros casuales o fortuitos. La violencia, al haber sido parte integral de la vida en sus familias, subyace en su actuar y surge ante cualquier provocación, acompañada de un conjunto de creencias y valores negativos sobre las relaciones con otros que legitima su uso como método válido de interacción.

"En mi caso, yo me considero violenta, no a golpes, no golpear a la gente, pero así, indiscriminadamente, soy muy hiriente como mi mamá para decir las cosas, soy demasiado hiriente para decir las cosas, y no nada más con... o sea, agarro parejo ¿no? si voy en la calle y le hacen algo a mi pareja, me volteo y me vale un cacahuate quién seas, una vez me le puse a un policía... soy muy, muy hiriente, eso también es violencia, al fin y al cabo" (Jazmín, M, 25 años).

Cabe mencionar que al igual que en la pareja, no sólo se aprenden y replican conductas violentas sino aquellas relacionadas con pobres procesos comunicativos, con escasas habilidades para la identificación y con el manejo de emociones y la resolución de conflictos, que en conjunto dificultan el desarrollo de relaciones satisfactorias o enriquecedoras en cualquier contexto. 
"Porque yo mucho tiempo estuve reprimiendo muchas (emociones), no sólo con mi pareja, en amistades, en familia y hasta estos momentos, mmm... que podría decir, que las situaciones me sobrepasaron pues ya actué de una manera más agresiva que cualquier palabra ¿no?... entonces si dije wow, como que lo tuve que haber externado en su momento" (Ramón, H, 19 años).

\section{Discusión}

De manera coincidente con lo encontrado en estudios previos, los resultados de esta investigación nos permiten apuntar al impacto que tiene la exposición a modelos violentos de comportamiento en el hogar de origen, con el desarrollo de dinámicas violentas al interior de las parejas conformadas por personas del mismo sexo (López \& Ayala, 2011; Padilla, 2015). Si bien se trata de una realidad ampliamente estudiada en un contexto heterosexual (Yedra, González-Flores, Oliva-Zárate \& Rivera-Vargas, 2015), los hallazgos de este estudio permiten ampliar el panorama de los repetitivos mecanismos conflictuales a través de las generaciones, más allá del sexo o género asumido por los individuos involucrados.

El haber realizado este estudio tomando como base fundamental el discurso propio de los sujetos actuantes, nos permitió acercarnos a la experiencia actual de este grupo poblacional, que si bien comentó experimentar la violencia de manera similar a la población heterosexual, nos dio pautas para distinguir posibles líneas de investigación sobre las cuales se requeriría ahondar más; por ejemplo, si se posiciona como factor causal o predisponente propio de las personas gays, lesbianas y bisexuales para la perpetuación de la violencia, el impacto del rechazo por parte de la familia hacia su orientación sexual -documentado en estudios previos como el de Perucchi et al., en 2014que en dos de los casos llevó a la pérdida -en una de manera temporal y en otra definitivadel vínculo familiar, lo cual disminuyó el apoyo percibido del medio por las personas participantes al momento de hacer frente a una situación violenta.
Así mismo, sugerimos para futuros estudios identificar qué tanto los modelos heteronormados, no sólo en la familia de origen sino en las campañas preventivas y medios de comunicación, respecto de lo que es la violencia, generan en esta población cierto distanciamiento de la problemática, por asumirse distinta a lo que se les presenta visual y auditivamente. Aunque la información sobre la conciencia de estigma por ser gays, lesbianas o bisexuales, junto con otros estresores minoritarios, ya han sido estudiados como causales de la violencia en estas parejas (Carvalho et al., 2011; Marín, 2009), no encontramos en la literatura revisada el impacto que puede causar sobre el reconocimiento de la violencia en esta comunidad, el hecho de que sólo sea presentada en un contexto heteronormado, ya sea en la familia o en la sociedad.

Respecto a la experiencia de violencia en las relaciones de pareja de los individuos participantes, es notable que en el total de los casos manifiesten haber vivido o vivir actualmente algún tipo de violencia, aunque esto haya podido ser influenciado por la misma convocatoria al reclutar a las personas interesadas por la temática explícita de la investigación; sugerimos no perder de vista que la ocurrencia de la problemática en el total de los sujetos participantes es coincidente con los altos porcentajes de incidencia de ésta, según estudios previos que señalan que el $59 \%$, el $70 \%$, y hasta el $100 \%$ de la muestra estudiada en países como Chile, Puerto Rico, y México, sufrían algún tipo de embate (Marín, 2009; López-Ayala, 2011; Padilla, 2015; Reyes et al., 2005).

Si bien es cierto que se requería de estudios con datos más exactos sobre la ocurrencia de esta problemática en las relaciones de pareja del mismo sexo en México y Latinoamérica, estas cifras podrían indicar que actualmente esta realidad se presenta como un foco rojo a atender, especialmente si se considera que las víctimas están expuestas a lesiones que ponen en riesgo su vida, como en el caso de uno de los participantes de este estudio quien requirió de atención hospitalaria tras ser atacado por su pareja. 
Es necesario mencionar como principal limitante de este estudio que los resultados presentados no son generalizables a toda la población, puesto que las personas participantes formaron parte de una muestra no probabilística reclutada por conveniencia, en la que las variables -sociales, psicológicas y físicas- de cada participante que no fueron consideradas como criterios de inclusión o exclusión, pudieron influir en la naturaleza de los datos recabados durante los grupos focales.

\section{Lista de referencias}

Aldarte (2010). Estudio sobre violencia intragénero. Recuperado de: http://www. aldar te.org/comun/imagenes/documentos/ INFORMEENCUESTAVIOLENCIA INTRAGENERO.pdf

Bowen, M. (1991). De la familia al individuo. Barcelona: Paidós.

Canales, J. L. (2014). Padres tóxicos: legado disfuncional de una infancia. México, D. F.: Paidós.

Carvalho, A.; Lewis, R.; Derlega, V.; Windstead, A. \& Viggiano, C. (2011). Internalized sexual minority stressors and same-sex intimate partner violence. Family Violence, 26, pp. 501-509. Doi: 10.1007/s10896011-9384-2.

Castañeda, M. (2011). La experiencia homosexual: para comprender la homosexualidad desde dentro y desde fuera. México, D. F.: Paidós.

Delgado, J. \& Gutiérrez, J. (1995). Métodos y técnicas cualitativas de investigación en ciencias sociales. Madrid: Síntesis.

Escobar, J. \& Bonilla-Jiménez, F. (2009). Grupos focales: una guía conceptual y metodológica. Cuadernos Hispanoamericanos de Psicología, 9 (1), pp. 51-67. Recuperado de: https://issuu. com/universidadelbosque/docs/vol9

Estrada, L. (2014). El ciclo vital de la familia. México, D. F.: Debolsillo.

Gil, L.; Machado, C. \& Antunes, R. (2006). Violência nas relações homossexuais: a fase oculta da agressão na intimidade. Recuperado de: https://www.rea.pt/imgs/ uploads/doc-estudos-2009-violencia- relacoes-homossexuais-face-ocultaagressao-intimidade.pdf

López, M. \& Ayala, D. (2011). Intimidad y las múltiples manifestaciones de violencia doméstica entre mujeres lesbianas. Salud y sociedad, 2 (2), pp. 151-174. Recuperado de: http://dialnet.unirioja.es/servlet/ articulo? codigo $=3899554$

Mak, W. W. S.; Chong, E. S. K. \& Kwong, M. M. F. (2010). Prevalence of same-sex intimate partner violence in Hong Kong. Public Health, 1 (24), p. 149-152. Doi: 10.1016/j.puhe.2010.02.002.

Marín,A. (2009). Maltrato y violencia al interior de las relaciones de pareja lesbianas: el segundo closet. Tesis de licenciatura, Universidad de Chile, Santiago de Chile, Chile. Recuperado de: http://www.tesis. uchile.cl/handle/2250/106198

Molina, R.; Echeverría, G. \& Henseleit, R. (2014). Violencia intragénero entre parejas de lesbianas entre los 25 y 29 años. Tesis de licenciatura, Universidad academia de humanismo cristiano, Chile. Recuperado de:

http://bibliotecadigital.academia.cl/ bitstream/handle/123456789/1757/ TPSICO\%20578.pdf?sequence=1

Onwuegbuzie, A. J.; Dickinson, W. B.; Leech, N. L. \& Zoran, A. G. (2011). Un marco cualitativo para la recolección y análisis de datos en la investigación basada en grupos focales. Paradigmas, 3, pp. 127-157. Recuperado de: https://dialnet.un irioja .es/ descarga/articulo/3798215.pdf

Padilla, P. (2015). La violencia no discrimina: relaciones lésbicas. I coloquio de investigación en género desde el IPN. Memorias, 1 (1), pp. 411-428. Recuperado de: www.genero.ipn.mx/Difusion/ Documents/mtc26.pdf

Perucchi, J.; Coelho, B. \& Dos Santos, H. I. (2014). Aspectos psicossociais da homofobia intrafamiliar e saúde de jovens lésbicas e gays. Estudios de Psicología, 19 (1), pp. 67-76. Doi:10.1590/S1413$294 X 201400010009$.

Prieto-Montoya, J. A.; Cardona-Castañeda, L. M. \& Vélez-Álvarez, C. (2016). Estilos parentales y consumo de sustancias 
psicoactivas en estudiantes de $8^{\circ}$ a $10^{\circ}$. Revista Latinoamericana de Ciencias Sociales, Niñez y Juventud, 14 (2), pp. 1345-1356. Doi:10.11600/169271 $5 x .14231161015$.

Quilaqueo, D. \& San Martín D. (2008). Categorización de saberes educativos mapuche mediante la teoría fundamentada. Estudios Pedagógicos. 34 (2), pp. 151-168. Doi: 10.4067/S0718-07052008000200009.

Reyes, F. R.; Rodríguez, J. R. \& Malavé, S. (2005). Manifestaciones de la violencia doméstica en una muestra de hombres homosexuales y mujeres lesbianas puertorriqueñas. Revista Interamericana de Psicología, 39 (3), pp. 449-456. Recuperado de: https:/dialnet.unirioja.es/ servlet/articulo? codigo $=2990435$

Rubio, M. \& Varas, J. (2004). El análisis de la realidad en la intervención social: métodos y técnicas de investigación. Madrid: CCS.

Segura, G.; Gil, M. \& Sepúlveda, M. V. (2006). El síndrome de alienación parental: una forma de maltrato infantil. Cuadernos de Medicina Forense, 12 (43-44), pp. 117128. Recuperado de: http://scielo.isciii.es/ $\mathrm{pdf} / \mathrm{cmf} / \mathrm{n} 43-44 / 09 . p d f$

Toro-Alfonso, J. \& Rodríguez-Madera, S. (2003). Violencia doméstica en parejas de hombres gay puertorriqueños, prevalencia, violencia intergeneracional, conductas adictivas $y$ destrezas de manejo de conflictos. Recuperado de: http://pepsic.b vsalud.org/pdf/pp/v3-4/v3-4a18.pdf

Vargas, R. (2003). Pétalos y espinas: hombres gay, relaciones de pareja y violencia. Buenos Aires: Elaleph.com

Yedra, L. R.; González-Flores, M. P.; OlivaZárate, L. \& Rivera-Vargas, E. A. (2015). Violencia psicológica en los noviazgos de adolescentes y su relación con las formas de interacción de sus padres. Recuperado de:

https://www.researchgate.net/ publication/292157132_Violencia psicologica_en_los_noviazgos_de adolescentes_y_su_rēlacion_con_las_formas_de_interaccion_de_sus_padres 